\title{
Study on the Application Comparison of Reactive Power Compensation Self-control Scheme in Power Design
}

\section{Lin Qiao}

Yunnan University of Business Management, Kunming, Yunnan, 650106

\author{
Keywords: Reactive Power Compensation, Self-control Scheme, Power Design
}

\begin{abstract}
The power system is closely related to social production and life. China's electricity demand is growing rapidly, the power grid is more complicated, and the demand for reactive power is increasing. It is necessary to install reactive power compensation devices in the power grid to meet power demand. In the power reactive compensation scheme, the active power filter adopts an ideal reactive power compensation method, which can compensate for the reactive power and harmonics of the change, reflect the operating status of the power grid system in real time, complete human-computer interaction, and realize automatic control, providing protection for the safe operation of the system through fault alarms.
\end{abstract}

\section{Introduction}

In recent years, China's national economy has developed rapidly, and more and more companies have adopted asynchronous transformers or asynchronous motors. However, it is because of the existence of high-power impact loads and thyristors. The use of this, in turn, causes the overall power factor of the power system to drop, resulting in a significant increase in voltage fluctuations. For this problem, the way we apply reactive compensation to it is mainly parallel capacitors, in order to improve the power factor, reduce power loss and ensure the quality of power supply. Especially when the transmission distance is long, the transmission capacity must be further improved to achieve a balance between the three-phase active power and the reactive power. Since the compensation methods used are not the same, the reactive power compensation can be roughly divided into three categories, namely, local compensation, distributed compensation, and centralized compensation. Among them, the general setting is concentrated in the local substation or the $6 \sim 35 \mathrm{kV}$ bus of the enterprise, and the compensation device usually includes the following aspects, such as parallel capacitors, static compensators, and synchronous cameras, etc. The fundamental purpose is to further change the grid power factor and compensate for reactive losses. However, usually the dispersion compensation device is installed in the high and low voltage bus of the village or the workshop with low power factor. This method has certain similarities with the centralized compensation method, but its reactive capacity is very small. The distributed compensation device is mainly used for $10 \mathrm{kV}$ parallel outdoor capacitors, and is set in the overhead line tower to effectively improve the power factor of the transmission network and ultimately achieve the purpose of power loss. Because this compensation method has many advantages, such as: small investment, high efficiency, simple maintenance, etc., it is often installed in long-distance transmission lines with low power factor and large load. In addition, the local compensation method is mainly installed around the asynchronous motor or the inductive equipment, so that not only can the power factor of the grid be improved, but also the quality of the power supply voltage of the equipment can be improved. However, it is clearly stated in the "Code for Design of Power Supply Systems" that has been promulgated and implemented by the state: in buildings, low-voltage capacitors should be dispersed. Therefore, when the motor is installed in a company or a factory or mine, local reactive compensation must be performed. In addition, combined with different compensation control methods, we can be divided into a variety of, such as: electronic, microcontroller control, PLC control. Therefore, in the power design process, it must be ensured that the selected reactive power compensation automatic control scheme is scientific and reasonable. This is of great significance for improving power supply reliability and reducing power loss. 


\section{Classification of reactive power compensation control scheme design}

The electronic automatic compensation control scheme design is composed of multiple discrete components. The automatic control system composed of discrete components mainly involves the following parts, such as phase, current detecting unit, reactive power calculation, capacitor, switching unit and so on. However, such compensation control schemes have many shortcomings, such as: many components, large volume, complicated line maintenance, and short service life. In addition, some companies only perform manual control because they cannot repair the equipment in time.

MCU control technology. Generally speaking, the system consists of a number of modules, such as a keyboard control module, a signal conditioning module, a display module, and a control compensation module. Because it has a single-time cycle execution instruction time and advanced instruction set, ATmega16 has a data throughput rate of $1 \mathrm{MIPS} / \mathrm{MHz}$, which can solve the contradiction between processing speed and power consumption. In addition, in the core of the ATmega16 microcontroller, there are very rich instructions, and there are 32 working registers. At the same time, these logic units and registers are connected to each other, so that the instructions can access two different registers simultaneously in one clock cycle. This structural device can greatly improve the code efficiency and has higher data throughput rate than ordinary CTSC microcontrollers.

The signal processing process of AVR is mainly as follows: (1) the signal from the A/D converter is sampled; 2 the collected signal is effectively processed by the FFT algorithm; 3 is checked and judged whether the voltage is overvoltage or undervoltage, Whether the current is a negative value or the like; 4 combined results determine whether the capacitor needs to be removed. The design of the system is basically a modular design, specifically involving four major modules, namely display and keyboard modules, data acquisition modules, capacitor switching modules and grid parameter calculation modules. The system is connected and debugged by modifying the program. At this stage, the mine WBB explosion-proof automatic replenishing martial arts device that we often adopt is the core controller of the single-chip microcomputer, which is used to detect the current, voltage and reactive power of the entire power system to help compensate the reactive power. . The display module provided in the device is capable of displaying the capacitor switching state, reactive power, load current and power factor.

PLC is a new generation of industrial control devices based on microcomputer technology. The main circuit, phase angle detection circuit, output circuit and regulated power supply of the original system continue to be used, and the hardware circuits such as addition and subtraction level conversion and delay circuit, clock pulse generator, reversible counter, clear circuit, decoder, etc. The control function is implemented by PLC. Since the output signal of the phase angle detection circuit is weak, it is not enough to drive the input of the PLC, so the signal is amplified and processed as the input signal of the PLC. According to the control requirements of the system, the software of the PLC is used to realize automatic control. The triode switching circuit in the original output circuit is realized by the output relay of the PLC. Due to the limitation of the PLC output point capacity, an intermediate relay is added as an output circuit.

\section{Main technology of reactive power compensation automatic control in power supply system}

In the automation grid system control, the monitored data should be collected first, and the status information data, fault parameter data, etc. of the grid operation can be displayed through the acquisition device, including pulse, analog quantity, state quantity, protection action information, fixed value information, etc. . The more important state quantities are collected through the two-position contacts to ensure that the information can be accurately reflected. The state quantity data includes circuit breaker status information, isolation switch, transformer tap position, etc. The analog quantity acquisition mainly includes bus voltage sampling, power sampling, and return current sampling. The pulse quantity acquisition has data such as acquisition action signal, protection status, and fixed value, mainly relay protection data. Data acquisition requires data 
sampling and transformation processing. The sampled analog signal is converted, which causes errors, requires data analysis correction, digital filtering operation, FFI spectrum analysis, etc., and then outputs to the next module. After the sensor, the signal to be measured enters the system, requires pre-processing, adjusts the level ratio and other processes to meet the sampling requirements.

The active power filter can dynamically suppress the power supply harmonics generated by the power system and compensate the reactive power. Designing the reactive power compensation automatic control system of the power supply system, the hardware design is the basic framework, and the hardware design affects the stability of the system and the performance of the operation, thus affecting the monitoring effect and overall operation of the system. To realize the basic functions of automatic control, it is necessary to have good hardware circuits to provide a basis for the transformation and upgrading of the later systems. The reactive power compensation automatic control system requires real-time remote monitoring of the operation of the power grid, as well as high signal transmission speed, and can collect and process the field data in large quantities, and complete more equipment management work. In the design, high-end digital signal processor is needed, which is used in the control core system, has high control ability and signal processing capability, has high-performance DSP function and microcontroller function, performs complex control calculation and completes operation monitoring.

\section{Reactive power compensation automatic control scheme comparison}

It can be seen from the above analysis that the traditional electronic automatic compensation control scheme has slow response speed, complicated lines and poor reliability. The reactive power compensation self-control scheme based on single-chip microcomputer control technology has poor anti-interference ability, and the reliability in the field of medium and high voltage reactive power compensation is not easy to guarantee. In addition, the higher the voltage level, the larger the radiation range and the fault surface of the substation. The reactive power automatic compensation scheme based on PLC control technology has the advantages of convenient configuration, easy expansion, high reliability, strong anti-interference ability, simple circuit and convenient installation and maintenance. It is the preferred solution for power system design and complete reactive power compensation device.

\section{Conclusion}

Only when choosing a more optimized reactive power compensation design scheme can it be possible to greatly reduce the grid power factor and reduce the power loss. It can be said that this has important practical significance for the future power grid construction. Through analysis and comparison in this paper, we only need to grasp key technical indicators, such as reliability, economy, safety, etc., and then conduct in-depth research on the implementation of reactive power compensation schemes for power systems, so as to design for future power systems. Provide more valuable references. Therefore, in the power design process, it must be ensured that the selected reactive power compensation automatic control scheme is scientific and reasonable. This is of great significance for improving power supply reliability and reducing power loss. The author of this article, combined with his own years of practical experience, focused on the reasons, meanings and compensation methods of reactive power compensation. And the design structure and principle of various types of reactive power compensation control schemes are elaborated. At the same time, the performance and structural design characteristics of each scheme are analyzed and compared, which will provide more valuable reference for power design in the future. Enterprise development brings greater economic and social benefits.

\section{References}

[1] Pan Aiqiang. Discussion on the application of self-control scheme for reactive power 
compensation in smart grid power design [J]. Low Carbon World, 2015(24): 50-51.

[2] Zheng Zhiwei. Discussion on the application of reactive power compensation self-control scheme in power design [J]. China Science and Technology, 2015 (02): 179.

[3] Yang Jinbao. Design of wireless calling system based on nRF905 in hospital ward [J]. Enterprise Technology Development: Academic Edition, 2011, 30(11) : 11 - 13.

[4] Ma Jinxiang, He Yiming. Design of AT89S MCU wireless transceiver system based on nRF905 module [J]. Communication Technology, 2009, 42( 2) 36 - 38.

[5] Wang Ping, Chen Changqing, Gong Rui, et al. Wireless serial communication system based on nRF905 [J]. Microcomputer Information, 2007 (32): 281 - 282. 